\author{
${ }^{1}$ J. Jenis ${ }^{*},{ }^{1}$ G.B. Bazhikova, \\ ${ }^{1}$ M.A. Dusebaeva, ${ }^{1}$ Zh.A. Abilov, ${ }^{2,3}$ H. Aisa \\ ${ }^{1}$ Faculty of Chemistry and Chemical Technology, Al-Farabi Kazakh National University, \\ Almaty, Kazakhstan \\ ${ }^{2}$ Xinjiang Technical Institutes of Physics and Chemistry, \\ Central Asian of Drug Discovery and Development \\ ${ }^{3}$ Xinjiang Key Laboratory of Plant Resources and Natural Product Chemistry, \\ XTIPC CAS, R. P. China \\ *E-mail: janarjenis@mail.ru
}

\title{
The constituents of volatile oils from Thymus altaicus
}

\begin{abstract}
The volatile oil constitutes were extracted from the aerial parts of Thymus altaicus by water steam distillation were analyzed by GC-MS method. Fifty six compounds were separated. Their relative contents were determined by area normalization in which 47 volatiles were identified. The major volatile oils of $T$. altaicus are 3- cyclohexene-1-methanol, $\alpha, \alpha-4$-trimethyl- (35.84\%), benzene, 1-mehtyl-2-(1- methylethyl)(15.24\%), $\beta$-myrcene (10.30\%), thymol (7.94\%), 1,4-cyclohexadiene, 1-methyl-4-(1-methylethyl)(5.34\%), caryophyllene (5.26\%), 1,6-octadien-3-ol,3,7-dimethyl (4.78\%), 2,6-octadien-1-ol,3,7-dimethyl-, (E)- (2.06\%), 2,6-octadien-1-ol, 3,7-dimethyl-, acetate, (E)- (1.31\%), caryophyllene oxide (1.23\%).
\end{abstract}

Key words: Thymus altaicus; Altay region; Volatile oils; GC-MS;

\section{Introduction}

The traditionally natural products have played an important role in developing of natural product chemistry which continues to expand to exciting new frontiers of great importance in medicine. In Kazakhstan 49 genuses, 247 species of plants are growing that belong to family Lamiaceae and genus Thymus have around 27 species [1]. Thymus altaicus has efficiently been used in the treatments of cold and cough with the properties of immunostimulant, expectorant, diuretic and detumescence in Kazakh traditional medicine [2].

Essential oils (EO) are also known as volatile oils, which is a concentrated hydrophobic liquid containing volatile aroma compounds and internationally defined as the product obtained by hydrodistillation, steam distillation or dry distillation or by a suitable mechanical process without heating (for Citrus fruits) of a plant or of some parts of it [35]. Essential oils of aromatic plants and spices are used in industries for production of bath products, cosmetics, soap, perfumes and toiletries. Many of them are also used in traditional medicine for various purposes such as aromatherapy for the purpose of altering one's mood, cognitive, psychological and physical well-being [5].

In this study an attempt has been made to investigate the chemical constituents of volatile oils from Kazakh traditional medicinal plants of Thymus altaicus grown in Altay region of Kazakhstan for the first time.

\section{Materials and Methods}

Plant material: Thymus altaicus was collected in Altay region of east Kazakhstan, in July 2012 and identified by Prof. Shen Guan Min from the Xinjiang Institute of Ecology and Geography, Chinese Academy of Sciences. The oils were isolated by water-distillation for $4 \mathrm{hrs}$ and then dried over anhydrous sodium sulphate.

GC-MS analysis: the aerial part of Thymus altaicus were analyzed by Electron Impact Ionization (EI) method on Perkin-Elemer Autosystem XL -TurboMass (Gas Chromatograph coupled to 
Mass Spectrometer) fused silica capillary column (30m $\times 2.5 \mathrm{~mm} ; 0.25 \mu \mathrm{m}$ film thickness), coated with PE-5 ms were utilized. The carrier gas was helium (99.999\%). The column temperature was programmed from $60^{\circ} \mathrm{C}$ (held for $5 \mathrm{~min}$ ), at $2^{\circ} \mathrm{C} / \mathrm{min}$ to $180^{\circ} \mathrm{C}$, at $3.5^{\circ} \mathrm{C} / \mathrm{min}$ to $290^{\circ} \mathrm{C}$.

The latter temperature maintained for $40 \mathrm{~min}$ (Acquisition parameters full scan; scan range 40$350 \mathrm{amu}$ ). The injector temperature was $310^{\circ} \mathrm{C}$. Injection: with a 0.1ul: detector ion source (EI-70eV). Samples were injected by splitting with the split ratio 1:60.

Identification of the compounds: Identification of compounds was done by comparing the NIST and Wiley library data of the peaks and mass spectra of the peaks with those reported in literature. Percentage composition was computed from GC peak areas on PE-5 ms column without applying correction factors.

\section{Results and discussion}

Volatile oils from the aerial parts of Thymus altaicus were analyzed by GC-MS. Fifty six compounds were separated. Their relative contents were determined by area normalization. Obtained data are presented in Table 1. The yield from whole herbs of Thymus altaicus was found to be $5.53 \%$. Table 1 report the composition of the volatiles of the aerial parts of $T$. altaicus. Forty seven components have been identified in the volatiles of $T$. altaicus which the major constituents are 3-cyclohexene1-methanol, $\alpha, \alpha, 4$-trimethyl- (35.84\%), benzene, 1-mehtyl-2-(1- methylethyl)- (15.24\%), $\beta$-myrcene $(10.30 \%)$, thymol (7.94\%), 1,4-cyclohexadiene,1methyl- 4-(1-methylethyl)- (5.34\%), caryophyllene (5.26\%), 1,6-octadien- 3-ol,3,7-dimethyl- (4.78\%), 2,6-octadien-1-,3,7-dimethyl-, (E)- (2.06\%) 2,6-octadien-1-ol, 3,7-dimethyl-, acetate, (E)- (1.31\%), caryophyllene oxide $(1.23 \%)$. According to the report 3-cyclohexene-1-methanol, $\alpha, \alpha, 4$-trimethyl( $\alpha$-terpineol) (35.84\%) shows antioxidant effects and antiseptic is present in many extracted oils of various plant species, acts as an antihypernociception and anti-inflammatory [7]. And second major volatile constituent benzene, 1-mehtyl-2-(1- methylethyl)- (15.24\%) showed antimicrobial and antibacterial activates $[8,9]$. $\beta$-myrcene $(10.30 \%)$ and essential oils containing this terpenoid compound are widely used as a fragrance in cosmetics, as a scent in household products, and as a flavoring additive in food and alcoholic beverages [10]. Furthermore, it was reported that $\beta$-myrcene is an analgesic substance and the active principle of lemongrass (Cymbopogon citratus Stapf) 'abafado', an infusion made with the pan covered in order to prevent the loss of volatile constituents [11]. Lemongrass 'abafado' is widely used in Brazilian folk medicine as a sedative and as a remedy for gastrointestinal disorders [12].

Table 1 - The volatile constituents of aerial parts of Thymus altaicus

\begin{tabular}{|c|l|c|c|c|c|}
\hline $\begin{array}{c}\text { Peak } \\
\text { No. }\end{array}$ & \multicolumn{1}{|c|}{ Constituents } & $\mathrm{t}_{\mathrm{R}}(\mathrm{min})$ & Molecular Formula & MW & $\begin{array}{c}\text { Content } \\
(\%)\end{array}$ \\
\hline 1 & 1 -R, $\alpha$ & 16.03 & $\mathrm{C}_{10} \mathrm{H}_{16}$ & 136 & 0.72 \\
\hline 2 & Bicyclo[3.1.0]hex-2-ene, 2-methyl-5-(1-methylethyl) & 16.17 & $\mathrm{C}_{10} \mathrm{H}_{16}$ & 136 & 0.32 \\
\hline 3 & Camphene & 18.46 & $\mathrm{C}_{10} \mathrm{H}_{16}$ & 136 & 0.77 \\
\hline 4 & $\beta$-Pinene & 20.96 & $\mathrm{C}_{10} \mathrm{H}_{16}$ & 136 & 0.20 \\
\hline 5 & Bicyclo[3.1.0]hexene, 4-methylene-1-(1-methylethyl) & 21.81 & $\mathrm{C}_{10} \mathrm{H}_{16}$ & 136 & 0.60 \\
\hline 6 & 3-Myrcene & 24.92 & $\mathrm{C}_{10} \mathrm{H}_{16}$ & 136 & 10.30 \\
\hline 7 & Cyclohexene, 1-methyl-4-(1-methylethylidene) & 26.10 & $\mathrm{C}_{10} \mathrm{H}_{16}$ & 136 & 0.45 \\
\hline 8 & 2,3-Dehydro-1,8-cineole & 26.93 & $\mathrm{C}_{10} \mathrm{H}_{16} \mathrm{O}$ & 152 & 0.09 \\
\hline 9 & Limonene & 27.56 & $\mathrm{C}_{10} \mathrm{H}_{16}$ & 136 & 0.42 \\
\hline 10 & Eucalyptol & 28.43 & $\mathrm{C}_{10} \mathrm{H}_{18} \mathrm{O}$ & 154 & 0.66 \\
\hline 11 & 1,4-Cyclohexadiene,1-methyl-4-(1-methylethyl)- & 31.66 & $\mathrm{C}_{10} \mathrm{H}_{16}$ & 136 & 5.34 \\
\hline 12 & Benzene, 1-methyl-2-(1-methylethyl)- & 34.10 & $\mathrm{C}_{10} \mathrm{H}_{14}$ & 134 & 15.24 \\
\hline 13 & Bicyclo[4.1.0]hepta-2-ene, 3,7,7-trimethyl & 34.63 & $\mathrm{C}_{10} \mathrm{H}_{16}$ & 136 & 0.05 \\
\hline 14 & 1-Octen-3-ol & 48.93 & $\mathrm{C}_{8} \mathrm{H}_{16} \mathrm{O}$ & 128 & 0.31 \\
\hline 15 & Terpineol, Z-, beta- & 50.54 & $\mathrm{C}_{10} \mathrm{H}_{18} \mathrm{O}$ & 154 & 0.05 \\
\hline
\end{tabular}




\begin{tabular}{|c|c|c|c|c|c|}
\hline $\begin{array}{l}\text { Peak } \\
\text { No. }\end{array}$ & Constituents & $\mathrm{t}_{\mathrm{R}}(\min )$ & Molecular Formula & MW & $\begin{array}{c}\text { Content } \\
(\%)\end{array}$ \\
\hline 16 & Camphor & 56.01 & $\mathrm{C}_{10} \mathrm{H}_{16} \mathrm{O}$ & 152 & 0.58 \\
\hline 17 & 1,6-Octadien-3-ol, 3,7-dimethyl & 57.82 & $\mathrm{C}_{10} \mathrm{H}_{18} \mathrm{O}$ & 154 & 4.78 \\
\hline 18 & 1,6-Octadien-3-ol, 3,7-dimethyl-, acetate & 58.84 & $\mathrm{C}_{12} \mathrm{H}_{20} \mathrm{O}$ & 196 & 0.91 \\
\hline 19 & Germacrene d & 60.75 & $\mathrm{C}_{15} \mathrm{H}_{24}$ & 204 & 0.03 \\
\hline 20 & Bornyl acetate- & 61.27 & $\mathrm{C}_{12} \mathrm{H}_{20} \mathrm{O}_{2}$ & 196 & 0.16 \\
\hline 21 & Benzene, 2-methoxy-4-methyl-1-(1-methylethyl)- & 62.14 & $\mathrm{C}_{11} \mathrm{H}_{16} \mathrm{O}$ & 164 & 0.10 \\
\hline 22 & Caryophyllene & 63.13 & $\mathrm{C}_{15} \mathrm{H}_{24}$ & 204 & 5.26 \\
\hline 23 & 2-Methyl-6-methylene-oct-3,7-dien-2-ol & 64.16 & $\mathrm{C}_{10} \mathrm{H}_{16} \mathrm{O}$ & 152 & 0.04 \\
\hline 24 & Pulegone & 67.17 & $\mathrm{C}_{10} \mathrm{H}_{16} \mathrm{O}$ & 152 & 0.16 \\
\hline 25 & $\alpha$ & 69.10 & $\mathrm{C}_{15} \mathrm{H}_{24}$ & 204 & 0.29 \\
\hline 26 & 2-Octadienal, 3,7-dimethyl-, (Z)- & 69.89 & $\mathrm{C}_{10} \mathrm{H}_{16} \mathrm{O}$ & 152 & 0.22 \\
\hline 27 & 3-Cyclohexene-1-methanol- $\alpha$ & 71.40 & $\mathrm{C}_{10} \mathrm{H}_{18} \mathrm{O}$ & 154 & 35.84 \\
\hline 28 & Naphthalene, 1,2,34,4A,5,6,8A-octahydro-7-methyl & 72.74 & $\mathrm{C}_{15} \mathrm{H}_{24}$ & 204 & 0.11 \\
\hline 29 & 2,6-Octadien-1-ol, 3,7-dimethyl-, acetate, (Z)- & 73.14 & $\mathrm{C}_{12} \mathrm{H}_{20} \mathrm{O}_{2}$ & 196 & 0.10 \\
\hline 30 & 2,6-Octadienal, 3,7-dimethyl-, (E)- & 74.06 & $\mathrm{C}_{10} \mathrm{H}_{16} \mathrm{O}$ & 152 & 2.06 \\
\hline 31 & 2,6-Octadien-1-ol, 3,7-dimethyl-, acetate, (E)- & 75.57 & $\mathrm{C}_{12} \mathrm{H}_{20} \mathrm{O}_{2}$ & 196 & 1.31 \\
\hline 32 & 2,6-Octadienal, 3,7-dimethyl-, (Z)- & 78.86 & $\mathrm{C}_{10} \mathrm{H}_{16} \mathrm{O}$ & 152 & 0.80 \\
\hline 33 & 2,6-Octadien-1-ol, 3,7-dimethyl-, (E)- & 82.50 & $\mathrm{C}_{10} \mathrm{H}_{18} \mathrm{O}$ & 154 & 0.91 \\
\hline 34 & Benzenemethanol, $\alpha$ & 82.87 & $\mathrm{C}_{10} \mathrm{H}_{14} \mathrm{O}$ & 150 & 0.12 \\
\hline 35 & Cis-Z- $\alpha$ & 89.47 & $\mathrm{C}_{15} \mathrm{H}_{24} \mathrm{O}$ & 220 & 0.32 \\
\hline 36 & Trans-Z- $\alpha$ & 89.76 & $\mathrm{C}_{15} \mathrm{H}_{24} \mathrm{O}$ & 220 & 0.12 \\
\hline 37 & Caryophyllene oxide & 94.18 & $\mathrm{C}_{15} \mathrm{H}_{24} \mathrm{O}$ & 220 & 1.23 \\
\hline 38 & $\begin{array}{l}\text { 12-Oxabicyclo[9.1.0]dodeca-3,7-diene, } \\
\text { 1,5,5,8-tetramethyl }\end{array}$ & 98.20 & $\mathrm{C}_{15} \mathrm{H}_{24} \mathrm{O}$ & 220 & 0.05 \\
\hline 39 & Cuminol & 101.6 & $\mathrm{C}_{15} \mathrm{H}_{14} \mathrm{O}$ & 150 & 0.71 \\
\hline 40 & Thymol & 107.0 & $\mathrm{C}_{10} \mathrm{H}_{14} \mathrm{O}$ & 150 & 0.62 \\
\hline 41 & Tau-muurolol & 107.8 & $\mathrm{C}_{15} \mathrm{H}_{26} \mathrm{O}$ & 222 & 0.15 \\
\hline 42 & $\alpha$ & 108.4 & $\mathrm{C}_{15} \mathrm{H}_{24}$ & 204 & 0.02 \\
\hline 43 & Phenol, 2-methyl-5-(1-methylethyl)- & 109.0 & $\mathrm{C}_{10} \mathrm{H}_{4} \mathrm{O}$ & 150 & 0.27 \\
\hline 44 & $\alpha$ & 110.8 & $\mathrm{C}_{15} \mathrm{H}_{26} \mathrm{O}$ & 222 & 0.39 \\
\hline 45 & Tetracyclo[6.3.2.0(2,5).0(1,8)]tridecan-9-ol, 4,4-dimethyl & 115.3 & $\mathrm{C}_{15} \mathrm{H}_{24} \mathrm{O}$ & 220 & 0.21 \\
\hline 46 & 1H-3A,7-methanoazulene, octahydro-1,4,9,9-tetramethyl & 117.6 & $\mathrm{C}_{15} \mathrm{H}_{26}$ & 206 & 0.08 \\
\hline 47 & Aromadendrene oxide-(2) & 120.2 & $\mathrm{C}_{15} \mathrm{H}_{24} \mathrm{O}$ & 220 & 0.23 \\
\hline
\end{tabular}

\section{Conclusion}

The volatile oils constitutes were extracted from the aerial parts of Thymus altaicus by water steam distillation which analyzed by GC-MS method. Fifty six compounds were separated. Their relative contents were determined by area normalization in which 47 volatiles were identified. Active principles of the Kazakh traditional medicinal plant (T. altaicus) that responsible for the activity were determined.
While the major volatile constituents are 3 - cyclohexene-1-methanol, $\alpha, \alpha$, 4-trimethyl- (35.84\%), benzene, 1-mehtyl-2-(1- methylethyl)- (15.24\%), and $\beta$-myrcene $(10.30 \%)$ which possessing antihypernociception, anti-inflammatory, antimicrobial, antibacterial and analgesic activities separately.

Acknowledgement. This research was supported by the Chinese Academy of Sciences Visiting Fellowship for Researchers from Developing Countries (Grant No. 2013FFGB0003). 


\section{References}

1. Flora Kazakhstan, Almaty, 1963. - Vol.3. P. 204-213.

2. Xu X., Konirbay B., Jenis J., etal. The Kazakh Materia Medica, The Ethnic Press: Beijing. 2009. - P. 357.

3. P. Rubiolo, B. Sgorbini, E. Librto, C. Cordero, C. Bicchi. Essential oils and volatiles: sample preparation and analysis// Flavour Fragr. J. 2010.Vol. 25. - P. 282-290.

4. Bergström G., Rothschild M., Groth I., Crighton C. Oviposition by butterflies on young leaves: investigation of leaf volatiles// Chemoecology. 1994. - Vol. 5. - P. 147-158.

5. Kesselmeier J., Staudt M. Biogenic volatile organic compounds (VOC): An overview on emission, physiology and ecology// Journal of Atmospheric Chemistry. - 1999. - 33(1). - P. 23-88.

6. Jenis J., Burasheva G.Sh., Aisa H., Ba-hang, Abilov Zh.A. Constitutes of the Volatiles from

Atriplex tatarica // Nat. Prod. Res. Dev. - 2010. - 22 (6). - P.1040-1044.

7. Miguel M.G. Antioxidant and anti-inflammatory activities of essential oils: a short review// Molecules. - 2010. - Vol.15. - P. 9252-9287.
8. Cosge B., Turker A., Ipek A., Gurbuz B., Arslan N. Chemical compositions and antibacterial activities of the essential oils from aerial parts and corollas of Origanum acutidens (Hand.-Mazz.) Ietswaart, an Endemic Species to Turkey// Molecules. - 2009. - Vol.14: - P. 1702-1712.

9. Mahboubi M., Kazempour N. Chemical composition and antimicrobial activity of Satureja hortensis and Trachyspermum copticum essential oil// Iran. J. Microbiol. - 2011. - 3(4). - P. 194-200.

10. Leung A.Y. Encyclopedia of Common Natural Ingredients Used in Food, Drugs and Cosmetics// John Wiley \& Sons, 1980, New York.

11. Lorenzetti B.B., Souza G.P., Sarti S. J., Santos-Filho D. \& Ferreira S.H. Myrcene mimics the peripheral analgesic activity of lemongrass tea// Journal of Ethnopharmacology. - 1991. - 34. - P. 43-48.

12. Carlini E.A., Contar J.D., Silva-Filho A.R., Silveira-Filho N.G., Frochtengartten M.L. \& Bueno O.A. Pharmacology of lemongrass (Cymbopogon citratus Stapf.). I. Effects of teas prepared from the leaves on laboratory animals// Journal of Ethnopharmacology. - 1986. - Vol. 17. - P. 37-64. 